\title{
The Geometric Structure of Nonholonomic Mechanics
}

\author{
Wang Sang Koon \\ Control and Dynamical Systems \\ California Institute of Technology 116-81 \\ Pasadena, CA 91125 \\ koon@cds.caltech.edu
}

\begin{abstract}
Many important problems in multibody dynamics, the dynamics of wheeled vehicles and motion generation, involve nonholonomic mechanics. Many of these systems have symmetry, such as the group of Euclidean motions in the plane or in space and this symmetry plays an important role in the theory.

Despite considerable advances on both Hamiltonian and Lagrangian sides of the theory, there remains much to do. We report on progress on two of these fronts. The first is a Poisson description of the equations that is equivalent to those given by Lagrangian reduction, and second, a deeper understanding of holonomy for such systems. These results promise to lead to further progress on the stability issues and on locomotion generation.
\end{abstract}

\section{Symplectic and Poisson Geometry of Nonholonomic Systems}

Bloch, Krishnaprasad, Marsden and Murray [1996], hereafter denoted [BKMM], applied methods of geometric mechanics to the Lagrange-d'Alembert formulation and extended the use of connections and momentum maps associated with a given symmetry group to this case. The resulting framework, including the nonholonomic momentum and nonholonomic mechanical connection, provides a setting for studying nonholonomic mechanical control systems that may have a nontrivial evolution of their nonholonomic momentum.

The setting is a configuration space $Q$ with a (nonintegrable) distribution $\mathcal{D} \subset T Q$ describing the constraints. For simplicity, we consider only homogeneous velocity constraints. We are given a Lagrangian $L$ on $T Q$ and a Lie group $G$ acting on the configuration space that leaves the constraints and the Lagrangian invariant. In many example, the group encodes position and orientation information. For example, for the snakeboard, the group is $S E(2)$ of rotations and translations in the plane. The quotient space $Q / G$ is called shape space.

\footnotetext{
${ }^{1}$ Research partially supported by the DOE contract DEFG0395-ER25251.
}

\author{
Jerrold E. Marsden ${ }^{1}$ \\ Control and Dynamical Systems \\ California Institute of Technology 116-81 \\ Pasadena, CA 91125 \\ marsden@cds.caltech.edu
}

The dynamics of such a system is described by a set of equations of the following form:

$$
\begin{aligned}
g^{-1} \dot{g} & =-\mathcal{A}^{\mathrm{nh}}(r) \dot{r}+I^{-1}(r) p \\
\dot{p} & =\dot{r}^{T} H(r) \dot{r}+\dot{r}^{T} K(r) p+p^{T} D(r) p \\
M(r) \ddot{r} & =\delta(r, \dot{r}, p)+\tau
\end{aligned}
$$

The first equation is a reconstruction equation for a group element $g$, the second is an equation for the nonholonomic momentum $p$ (not conserved in general), and the third are equations of motion for the reduced variables $r$ which describe the "shape" of the system. The momentum equation is bilinear in $(\dot{r}, p)$. The variable $\tau$ represents the external forces applied to the system, and is assumed to affect only the shape variables, i.e., the external forces are $G$-invariant. Note that the evolution of the momentum $p$ and the shape $r$ decouple from the group variables.

This framework has been very useful for studying controllability, gait selection and locomotion for systems such as the snakeboard. It has also helped in the study of optimality of certain gaits, by using optimal control ideas in the context of nonholonomic mechanics (Koon and Marsden [1997a] and Ostrowski, Desai and Kumar [1997]). Hence, it is natural to explore ways for developing similar procedures on the Hamiltonian side.

\subsection{Symplectic Reduction}

Bates and Sniatycki [1993], hereafter denoted [BS], developed the symplectic geometry on the Hamiltonian side of nonholonomic systems, while [BKMM] explored the Lagrangian side. It was not obvious how these two approaches were equivalent, especially how the momentum equation, the reduced Lagrange-d'Alembert equations and the reconstruction equation correspond to the developments in [BS].

Our first main result establishes the specific links between these two sides and uses the ideas and results of each to shed light on the other, deepening our understanding of both points of view. For example, in proving the equivalence of the Lagrangian reduction and the symplectic reduction, we have shown where the momentum equation is lurking on the Hamiltonian side and how this is related to the organization of the dynamics of nonholonomic systems with symmetry into the three parts displayed above: a reconstruction equation for 
the group element $g$, an equation for the nonholonomic momentum $p$ and the reduced Hamilton equations for the shape variables $r, p_{r}$.

The basic theory is illustrated with the snakeboard, as well as a simplified model of the bicycle (see Getz and Marsden [1995]). The latter is an important prototype control system because it is an underactuated balance system. For more details, see Koon and Marsden [1997b].

\subsection{Poisson Geometry}

On the Hamiltonian side, besides the symplectic point of view, one can also develop the Poisson point of view. Because of the momentum equation, it is natural to let the value of momentum be a variable and for this a Poisson rather than a symplectic viewpoint is more natural. Some of this theory has been started in van der Schaft and Maschke [1994], hereafter denoted [VM]. In our second main result, we build on their work and develop the Poisson reduction for the nonholonomic systems with symmetry. We use this Poisson reduction procedure to obtain specific formulas for the nonholonomic Hamiltonian dynamics. We also show that the equations given by Poisson reduction are equivalent to those given by the Lagrangian reduction via a reduced constrained Legendre transform.

Two interesting complications make this effort especially interesting. First of all, as we have mentioned, symmetry need not lead to conservation laws but rather to a momentum equation. Second, the natural Poisson bracket fails to satisfy the Jacobi identity. In fact, the so-called Jacobiizer (the cyclic sum that vanishes when the Jacobi identity holds) is an interesting expression involving the curvature of the underlying distribution describing the nonholonomic constraints. We shall explore these in detail in Koon and Marsden [1997c].

Van der Schaft and Maschke [1994] use the Legendre transformation $\mathbb{F L}: T Q \rightarrow T^{*} Q$ to define the Hamiltonian $H$ in the standard fashion: $H=p_{i} \dot{q}^{i}-L$, where $p=\mathbb{F} L\left(v_{q}\right)=\partial L / \partial \dot{q}$, and then write the equations of motion in the Hamiltonian form as

$$
\left(\begin{array}{c}
\dot{q}^{i} \\
\dot{p}_{i}
\end{array}\right)=J\left(\begin{array}{c}
\frac{\partial H}{\partial \partial^{j}} \\
\frac{\partial H}{\partial p_{j}}
\end{array}\right)+\left(\begin{array}{c}
0 \\
\lambda_{a} \omega_{i}^{a}
\end{array}\right), \quad \omega_{i}^{a} \frac{\partial H}{\partial p_{i}}=0 .
$$

Here $J$ is the canonical Poisson tensor

$$
J=\left(\begin{array}{ll}
\left\{q^{i}, q^{j}\right\} & \left\{q^{i}, p_{j}\right\} \\
\left\{p_{i}, q^{j}\right\} & \left\{p_{i}, p_{j}\right\}
\end{array}\right)=\left(\begin{array}{cc}
0_{n} & I_{n} \\
-I_{n} & 0_{n}
\end{array}\right),
$$

where $\{$,$\} is the canonical Poisson bracket.$

On Lagrangian side, we saw that one can get rid of the Lagrangian multipliers. On the Hamiltonian side, it is also desirable to model the Hamiltonian equations without the Lagrange multipliers $\lambda$ by a vector field on a submanifold of $T^{*} Q$. In [VM], it is done through a clever change of coordinates.

First, a constraint phase space $\mathcal{M}=\mathbb{F} L(\mathcal{D}) \subset T^{*} Q$ is defined and in local coordinates,

$$
\mathcal{M}=\left\{(q, p) \in T^{*} Q \mid \omega_{i}^{a} \frac{\partial H}{\partial p_{i}}=0\right\} .
$$

Let $\left\{X_{\alpha}\right\}$ be a local basis for the constraint distribution $\mathcal{D}$ and let $\left\{\omega^{a}\right\}$ be a local basis for the annihilator $\mathcal{D}^{0}$. Let $\left\{\omega_{a}\right\}$ span the complementary subspace to $\mathcal{D}$ such that $\left\langle\omega^{a}, \omega_{b}\right\rangle=\delta_{b}^{a}$ where $\delta_{b}^{a}$ is the usual Kronecker delta. Here $a=1, \ldots, k$ and $\alpha=1, \ldots, n-k$. Define a coordinate transformation $(q, p) \rightarrow\left(q, \tilde{p}_{\alpha}, \tilde{p}_{a}\right)$ by

$$
\tilde{p}_{\alpha}=X_{\alpha}^{i} p_{i}, \quad \tilde{p}_{a}=\omega_{a}^{i} p_{i} .
$$

[VM] shows that in the new (generally not canonical) coordinates $\left(q, \tilde{p}_{\alpha}, \tilde{p}_{a}\right)$, the Poisson tensor becomes

$$
\tilde{J}(q, \tilde{p})=\left(\begin{array}{ll}
\left\{q^{i}, q^{j}\right\} & \left\{q^{i}, \tilde{p}_{j}\right\} \\
\left\{\tilde{p}_{i}, q^{j}\right\} & \left\{\tilde{p}_{i}, \tilde{p}_{j}\right\}
\end{array}\right) .
$$

and the constrained Hamiltonian equations transform into

$$
\left(\begin{array}{c}
\dot{q}^{i} \\
\tilde{\tilde{p}}_{\alpha} \\
\dot{\tilde{p}}_{a}
\end{array}\right)=\tilde{J}\left(\begin{array}{c}
\frac{\partial \tilde{H}}{\partial q^{j}} \\
\frac{\partial \tilde{H}}{\partial \tilde{p}_{\beta}} \\
\frac{\partial H}{\partial \tilde{p}_{b}}
\end{array}\right)+\left(\begin{array}{c}
0 \\
0 \\
\lambda_{b} K_{a}^{b}(q)
\end{array}\right), \quad \frac{\partial \tilde{H}}{\partial \tilde{p}_{a}}=0
$$

where $\tilde{H}(q, \tilde{p})$ is the Hamiltonian $H(q, p)$ expressed in the new coordinates $(q, \tilde{p})$.

Then, let $\left(\tilde{p}_{\alpha}, \tilde{p}_{a}\right)$ satisfy the constraint equations $\frac{\partial \tilde{H}}{\partial \tilde{p}_{\alpha}}(q, \tilde{p})=0$. Since

$$
\mathcal{M}=\left\{\begin{array}{l|l}
\left(q, \tilde{p}_{\alpha}, \tilde{p}_{a}\right) \mid \frac{\partial \tilde{H}}{\partial \tilde{p}_{a}}\left(q, \tilde{p}_{\alpha}, \tilde{p}_{a}\right)=0
\end{array}\right\},
$$

[VM] uses $\left(q, \tilde{p}_{\alpha}\right)$ as an induced local coordinates for $\mathcal{M}$.

Now we are ready to eliminate the Lagrange multipliers. Notice that $\frac{\partial \tilde{H}}{\partial \tilde{p}_{b}}(q, \tilde{p})=0$ on $\mathcal{M}$, and by restricting the dynamics on $\mathcal{M}$, we can disregard the last equations involving $\lambda$ in the above set of constrained Hamiltonian equations. In fact, we can also truncate the Poisson tensor $\tilde{J}$ by leaving out its last $k$ columns and last $k$ rows and then describe the constrained dynamics on $\mathcal{M}$ expressed in the induced coordinates $\left(q^{i}, \tilde{p}_{\alpha}\right)$ as follows

$$
\left(\begin{array}{c}
\dot{q}^{i} \\
\tilde{\tilde{p}}_{\alpha}
\end{array}\right)=J_{\mathcal{M}}\left(\begin{array}{c}
\frac{\partial H_{\mathcal{M}}}{\partial q^{j}}\left(q, \tilde{p}_{\alpha}\right) \\
\frac{\partial H_{\mathcal{M}}}{\partial \tilde{p}_{\beta}}\left(q, \tilde{p}_{\alpha}\right)
\end{array}\right), \quad\left(\begin{array}{c}
q^{i} \\
\tilde{p}_{\alpha}
\end{array}\right) \in \mathcal{M} .
$$

Here $J_{\mathcal{M}}$ is the $(2 n-k) \times(2 n-k)$ truncated matrix of $\tilde{J}$ restricted to $\mathcal{M}$ and is expressed in the induced coordinates. Also it is easy to show that

$$
\frac{\partial \tilde{H}}{\partial q^{j}}\left(q, \tilde{p}_{\alpha}, \tilde{p}_{a}\right)=\frac{\partial H_{\mathcal{M}}}{\partial q^{j}}\left(q, \tilde{p}_{\alpha}\right)
$$

and

$$
\frac{\partial \tilde{H}}{\partial \tilde{p}_{\beta}}\left(q, \tilde{p}_{\alpha}, \tilde{p}_{a}\right)=\frac{\partial H_{\mathcal{M}}}{\partial \tilde{p}_{\beta}}\left(q, \tilde{p}_{\alpha}\right),
$$


where $H_{\mathcal{M}}$ is the constrained Hamiltonian on $\mathcal{M}$ expressed in the induced coordinates.

The matrix $J_{\mathcal{M}}$ defines a bracket $\{,\}_{\mathcal{M}}$ on the constraint submanifold $\mathcal{M}$ as follows

$$
\begin{aligned}
& \left\{F_{\mathcal{M}}, G_{\mathcal{M}}\right\}_{\mathcal{M}}\left(q, \tilde{p}_{\alpha}\right):= \\
& \quad\left(\frac{\partial F_{\mathcal{M}}^{T}}{\partial q^{i}} \frac{\partial F_{\mathcal{M}}^{T}}{\partial \tilde{p}_{\alpha}}\right) J_{\mathcal{M}}\left(q^{i}, \tilde{p}_{\alpha}\right)\left(\begin{array}{c}
\frac{\partial G_{\mathcal{M}}}{\partial q^{j}} \\
\frac{\partial G_{\mathcal{M}}}{\partial \tilde{p}_{\beta}}
\end{array}\right)
\end{aligned}
$$

for any two smooth functions $F_{\mathcal{M}}, G_{\mathcal{M}}$ on the constraint submanifold $\mathcal{M}$. Clearly this bracket satisfies the first two defining properties of a Poisson bracket, namely, skew symmetry and Leibniz rule, and it is shown in [VM] that it satisfies the Jacobi identity if and only if the constraints are holonomic.

In Koon and Marsden [1997c], we will develop a general formula for the Jacobiizer which is an interesting expression involving the curvature of the underlying distribution that describes the nonholonomic constraints. From this formula, we can see clearly that the Poisson bracket defined here satisfies the Jacobi identity if and only if the constraints are holonomic.

\subsection{Poisson Reduction for Nonholonomic Me- chanics}

However, [VM] did not deal with the important case when the system have a symmetry group. In order to develop similar procedures for breaking the dynamics of such systems on the Hamiltonian side from a Poisson viewpoint, we need to build on their work and develop the Poisson reduction.

Let $G$ be the symmetry group of the system. We will write the equations of motion for the reduced constrained Hamiltonian dynamics using a reduced "Poisson" bracket on the reduced constraint phrase space $\overrightarrow{\mathcal{M}}$.

The crucial step here is how to represent the constraint distribution $\mathcal{D}$ in a way that is both intrinsic and ready for reduction. The work in both [BKMM] and Koon and Marsden [1997b] suggest that we should use the tools like nonholonomic momentum $p$ and the nonholonomic connection $\mathcal{A}$ in $[\mathrm{BKMM}]$ to describe $\mathcal{D}$

Recall that in $[\mathrm{BKMM}]$, a body fixed basis $e_{b}(g, r)=$ $\operatorname{Ad}_{g} \cdot e_{b}(r)$ has been constructed such that the infinitesimal generators $\left(e_{i}(g, r)\right)_{Q}$ of its first $m$ elements at a point $q$ span $\mathcal{S}_{q}=\mathcal{D}_{q} \cap T_{q}(\operatorname{Orb}(q))$ where $T_{q}(\operatorname{Orb}(q))$ is the tangent space to the orbit of the group. Assume that $G$ is a matrix group and $e_{i}^{d}$ is the component of $e_{i}(r)$ with respect to a fixed basis $\left\{b_{a}\right\}$ of the Lie algebra $g$ where $\left(b_{a}\right)_{Q}=\partial_{g^{a}}$, then $\left(e_{i}(g, r)\right)_{Q}=g_{d}^{a} e_{i}^{d} \partial_{g^{a}}$ Since $\mathcal{D}_{q}$ is the direct sum of $\mathcal{S}_{q}$ and the horizontal space of the nonholonomic connection $\mathcal{A}$, it can be represented by

$$
\mathcal{D}=\operatorname{span}\left\{g_{d}^{a} e_{i}^{d} \partial_{g^{a}},-g_{b}^{a} A_{\alpha}^{b} \partial_{g^{a}}+\partial_{r^{\alpha}}\right\} .
$$

Here $a, b, c, \ldots$ (corresponding to the symmetry direction) range from 1 to $k(k=\operatorname{dim} \mathfrak{g}) ; i, j, k, \ldots$ (symmetry direction along constraint space) range from 1 to $m ; \alpha, \beta, \ldots$ (shape variables $r$ ) range from 1 to $n-k$ $(n-k=\operatorname{dim}(Q / G))$.

Then the induced coordinates $\left(g^{a}, r^{\alpha}, \tilde{p}_{i}, \tilde{p}_{\alpha}\right)$ for the constraint submanifold $\mathcal{M}$ are defined by

$$
\begin{aligned}
\tilde{p}_{i} & =g_{d}^{a} e_{i}^{d} p_{a}=\mu_{d} e_{i}^{d} \\
\tilde{p}_{\alpha} & =p_{\alpha}-g_{b}^{a} A_{\alpha}^{b} p_{a}=p_{\alpha}-\mu_{b} A_{\alpha}^{b} .
\end{aligned}
$$

Here $\mu$ is an element of the dual of the Lie algebra $\mathfrak{g}^{*}$ and $\mu_{a}$ are its coordinates with respect to a fixed dual basis. Notice that $\tilde{p}_{i}$ are nothing but the corresponding momentum functions on the Hamiltonian side.

After applying the results of [VM] summarized in the previous section to these special induced coordinates and doing a reduction by the symmetry group $G$, we are able to prove the following theorem.

Theorem 1.1 The momentum equation and the reduced Hamilton equations on the reduced constraint submanifold $\overline{\mathcal{M}}$ can be written as follows

$$
\begin{aligned}
\dot{\tilde{p}}_{i} & =-\mu_{a} C_{b d}^{a} e_{i}^{b} e_{j}^{d} \frac{\partial h_{\overline{\mathcal{M}}}}{\partial \tilde{p}_{j}}+\mu_{a} F_{i \beta}^{a} \frac{\partial h_{\overline{\mathcal{M}}}}{\partial \tilde{p}_{\beta}} \\
\dot{r}^{\alpha} & =\frac{\partial h_{\overline{\mathcal{M}}}}{\partial \tilde{p}_{\alpha}} \\
\dot{\tilde{p}}_{\alpha} & =-\frac{\partial h_{\overline{\mathcal{M}}}}{\partial r^{\alpha}}-\mu_{a} F_{j \alpha}^{a} \frac{\partial h_{\overline{\mathcal{M}}}}{\partial \tilde{p}_{j}}-\mu_{a} B_{\alpha \beta}^{a} \frac{\partial h_{\overline{\mathcal{M}}}}{\partial \tilde{p}_{\beta}} .
\end{aligned}
$$

Here, $h_{\overline{\mathcal{M}}}$ is the reduced Hamiltonian on $\overline{\mathcal{M}} ; \xi=$ $\left(g^{-1}\right) \dot{g} ; C_{b d}^{a}$ are the structure coefficients of the Lie algebra $\mathfrak{g}$ and $F_{i \beta}^{a}$ is defined by

$$
F_{i \beta}^{a}=\frac{\partial e_{i}^{a}}{\partial r^{\beta}}+C_{b d}^{a} e_{i}^{b} A_{\beta}^{d} .
$$

With the following reconstruction equation

$$
\dot{\xi}^{b}=-A_{\beta}^{b} \frac{\partial h_{\overline{\mathcal{M}}}}{\partial \tilde{p}_{\beta}}+e_{i}^{b} \frac{\partial h_{\overline{\mathcal{M}}}}{\partial \tilde{p}_{i}},=-A_{\beta}^{b} \dot{r}^{\beta}+\left(I^{-1}\right)^{b i} p_{i}
$$

we recover the full dynamics of the system.

Notice that the first equation can be considered as the momentum equation on the Hamiltonian side which corresponds to the momentum equation developed in [BKMM]. It generalizes the Lie-Poisson equation to the nonholonomic case.

Furthermore, we have also proved

Theorem 1.2 The equations given by the Poisson reduction in Theorem 1 are equivalent to the equations given by the Lagrangian reduction

$$
\begin{aligned}
\xi^{b} & =-A_{\beta}^{b} \dot{r}^{\beta}+\left(I^{-1}\right)^{b i} p_{i}=-A_{\beta}^{b} \dot{r}^{\beta}+e_{i}^{b} \Omega^{i} \\
\dot{p}_{i} & =\frac{\partial l}{\partial \xi^{a}}\left(C_{b d}^{a} \xi^{b} e_{i}^{d}+\frac{\partial e_{i}^{a}}{\partial r^{\beta}} \dot{r}^{\beta}\right) \\
\frac{d}{d t}\left(\frac{\partial l_{c}}{\partial \dot{r}^{\alpha}}\right) & -\frac{\partial l_{c}}{\partial r^{\alpha}}=-\frac{\partial l}{\partial \xi^{b}}\left(B_{\alpha \beta}^{b} \dot{r}^{\beta}+F_{\alpha i}^{b} \Omega^{i}\right),
\end{aligned}
$$


via a reduced Legendre transform

$$
\tilde{p}_{\alpha}=\frac{\partial l_{c}}{\partial \dot{r}^{\alpha}} \quad \tilde{p}_{i}=\frac{\partial l_{c}}{\partial \Omega^{i}} .
$$

Here the reduced constrained Lagrangian is defined by $l_{c}(r, \dot{r}, \Omega)=l(r, \dot{r},-A \dot{r}+\Omega e)$. where $\Omega$ is the body angular velocity and $e(r)$ is the body fixed basis at the identity defined earlier.

Thus, we have developed the Poisson reduction and a corresponding procedure that can break the nonholonomic dynamics on the Hamiltonian side into a reconstruction equation, a momentum equation and the reduced Hamilton equations. Also we have shown that Hamiltonian reductions (Poisson and Symplectic) are equivalent to Lagrangian reduction. For more details, see Koon [1997].

These results are important for the future development of control as well as the stability theory for nonholonomic mechanical systems with symmetry. In particular, they will be required for the development of the powerful block diagonalization properties of the energy-momentum method developed by Simo, Lewis and Marsden [1991]. This technique is very important for the development of systematic methods for stability criteria.

\section{Holonomy and Locomotion.}

It is now well known how the geometric effect of holonomy plays an important role in the understanding of phase drifts and that this is a crucial ingredient in problems of stabilization and tracking. On the other hand, parts of the basic theory for this is not as well developed in the case of nonholonomic systems as for holonomic ones. We are specifically referring to the approach in which the holonomy is that associated with a connection that is constructed from the kinetic energy metric of the problem (the mechanical connection) and the constraints.

For systems with holonomic constraints, the geometric approach is described in Marsden, Montgomery and Ratiu [1990]. The basic idea of this approach is described below. On the other hand, these ideas are clearly important and are used for systems with nonholonomic constraints as well, as described in Marsden and Ostrowski [1997] and references therein.

The geometric tools needed to further develop the theory for systems with nonholonomic constraints are laid in Marsden, Montgomery and Ratiu [1990] and in [BKMM]. We aim to develop the theory by combining the approach in these two papers and also by making the calculations more concrete and accessible. In particular, in the latter paper the notion of the nonholonomic connection is defined and this is what replaces the mechanical connection in the case of holonomic constraints. As earlier, what makes this theory more interesting is the presence of the distribution describing the nonholonomic constraints (so that one gets a contribution of holonomy from two sources) as well as the fact that the momentum equation need not be conserved.

In [BKMM] it was also shown that the momentum equation is, in certain circumstances, a parallel transport equation, which introduces yet another connection. This structure is in fact very important in the stability theory of Zenkov, Bloch and Marsden [1997]. One can view the build up of monentum in locomotion problems (such as the snakeboard) as a holonomy effect itself, and this is coupled to the phase drifts in the configuration variables themselves.

\subsection{Bundles and Connection}

We now amplify a few basic facts on which the above locomotion ideas are based. For instance, consider the reorientation of a vehicle in space or an underwater vehicle due to the articulation of joints or due to the controlled spinning of internal rotors. Such reorientation can take place while the total system angular momentum is constant during the maneuver. This effect may be understood in terms of a division of variables for the entire configuration space $Q$ into group variables such as position and orientation variables and internal or shape variables such as rotor and joint angles. This gives rise to a principal bundle $\pi: Q \rightarrow S$ whose base space $S$ is the space of shape variables and whose fibers are just the group orbits.

The mechanical connection is a connection whose horizontal space is orthogonal (via the kinetic energy metric) to these fibers. A construction more familiar to mechanicians would be to compute the effective angular velocity of a system by multiplying the angular momentum by the inverse of the total system moment of inertia tensor. More precisely, the mechanical connection is a map $\mathcal{A}: T Q \rightarrow \mathfrak{g}$ that assigns to each $(q, v)$ the "angular velocity of the locked system"

$$
\mathcal{A}(q, v)=\mathbb{I}(q)^{-1} J(q, v) .
$$

Here $J$ is the momentum map. II $(q)$ is the locked inertia tensor which is the classical moment of inertia tensor of the instantaneous rigid system.

The mechanical connection plays a very important role in reduction theory (Marsden [1992]). Symmetry for mechanical systems often manifest themselves as invariances of the system dynamics with respect to translational or rotational inertial position. The goal of reduction theory is to factor out the invariances in order to provide a simplified analysis in terms of the base (shape) space. Observe motion in shape space alone is similar to watch the shapes change relative to an observer riding with the object. Then the problem of finding the original complete path is called reconstruction, which is closely linked. with the generation of geometric and dynamic phases.

For general holonomic mechanical system with symmetry, we can write down the information encoded by the mechanical connection in a very simple form. 
The conservation law can be written as $\mathcal{A}(q, v)=$ $\mathbb{I}(q)^{-1} J(q, v)=\mathbb{I}(q)^{-1} \mu$ where $\mu$ is a (constant) momentum. In a local trivialization, the conservation law becomes $\mathcal{A}(r, g)(\dot{r}, \dot{g})=\operatorname{Ad}_{g}\left(g^{-1} \dot{g}+\mathcal{A}_{\text {loc }}(r) \dot{r}\right)=$ $\left(\operatorname{Ad}_{g} \mathbb{I}_{\text {loc }}^{-1}(r) A d_{g}^{*}\right) \mu$. Rearranging this equation, we see that the group variables evolve according to

$$
\dot{g}=g\left(-\mathcal{A}_{\mathrm{loc}}(r) \dot{r}+\mathbb{I}_{\mathrm{loc}}^{-1}(r) p\right)
$$

where $p=A d_{g}^{*} \mu$ is the body angular momentum. Here, $\mathcal{A}_{\text {loc }}(r)$ and $\mathbb{I}_{\text {loc }}(r)$ are local forms of the mechanical connection and locked inertia tensor and will be abbreviated below by $A$ and $I$ respectively.

\subsection{Geometric and Dynamic Phases}

For concreteness, consider a simple example of two planar rigid bodies connected at their centers of mass by a pin joint. Let $I_{1}$ and $I_{2}$ be their moments of inertia and $\theta_{1}$ and $\theta_{2}$ be the angle they make with a fixed inertial direction. In this case, the shape space is the circle $S^{1}$ parametrized by the hinge angle $\psi=\theta_{2}-$ $\theta_{1}$ and the configuration space is $S^{1} \times S^{1}$ and can be parametrized by $(\theta, \psi)$ (if we set $\theta=\theta_{1}$ ). Conservation of angular momentum states that $I_{1} \dot{\theta}+I_{2}(\dot{\theta}+\dot{\psi})=\mu=$ constant; that is, $d \theta+\frac{I_{2}}{I_{1}+I_{2}} d \psi=\frac{\mu}{I_{1}+I_{2}} d t$. The left hand is the mechanical connection. Suppose that the total angular momentum is zero: $\mu=0$. When body 2 goes through one revolution so that $\psi$ traverses the base circle one time, body 1 rotates by the amount

$$
\Delta \theta=-\frac{I_{2}}{I_{1}+I_{2}} \int_{0}^{2 \pi} d \psi=-\left(\frac{I_{2}}{I_{1}+I_{2}}\right) 2 \pi,
$$

which is the total change in the group variable. But if the angular momentum is not zero, we will have

$$
\Delta \theta=-\frac{I_{2}}{I_{1}+I_{2}} \int_{0}^{2 \pi} d \psi+\frac{I_{2}}{I_{1}+I_{2}} \mu \int_{0}^{T} d t
$$

and equal to $\frac{I_{2}}{I_{1}+I_{2}}(-2 \pi+\mu T)$ where $T$ is the time body 2 takes to make one revolution.

One does not need the geometry of connections to understand such a simple example, but it does provide a simple illustration of the ideas. For more complex examples, the geometric setting of connections in general and the formula (2.2) in particular has indeed proven useful. Below we will mention some new results that make the calculations of the phases for dynamical systems on any trivial $S^{1}$-bundle more accessible. They also works for any $G_{\mu}$-bundle in the case $G_{\mu}=S^{1}$. Recall that $G_{\mu}$ is the isotropy subgroup of $G$ used for reduction: $J^{-1}(\mu) \rightarrow J^{-1} / G_{\mu}$. It is also used to construct the $G_{\mu}$-bundle: $Q \rightarrow Q / G_{\mu}$. In Marsden, Montgomery and Ratiu [1990], both of these two $G_{\mu^{-}}$bundle have played an important role in the study of the phases.

We have used these results to compute the phases for several well-known examples like planar $N$-body problem and the $3 \mathrm{D}$ rigid body. First let us state a result in Murray [1995] which describes a splitting of the reduced Lagrangian $l$ that enables one to write down the local forms of the locked inertia tensor and connection by directly looking at the reduced mass-inertial matrix.

Theorem 2.1 Let $L$ be G-invariant. The reduced Lagrangian can be written as

$$
l(r, \dot{r}, \xi)=\frac{1}{2}\left(\xi^{T}, \dot{r}^{T}\right)\left(\begin{array}{cc}
I & I A \\
A^{T} I & m(r)
\end{array}\right)\left(\begin{array}{c}
\xi \\
\dot{r}
\end{array}\right)-V(r)
$$

Theorem 2.2 For simple holonomic mechanical systems on a trivial $S^{1}$-bundle, the total phase $\Delta \theta$ of the reconstructed (integral) curve is given by

$$
\Delta \theta=-\oint A_{\alpha}(r) \dot{r}^{\alpha} d t+\mu \int_{0}^{T} I(r)^{-1} d t
$$

where the line integral is along $r(t)$ which is a closed (base integral) curve in the shape space with period $T$.

As we have mentioned, this result also holds for systems like the rigid body where $G_{\mu}=S^{1}$.

In case that the angular momentum is zero, if the system undergoes cyclic motion in the shape space, then it need not undergo cyclic motion in the configuration space. The difference between the beginning and the end of the motion is given by a drift in the group variables and this is the geometric phase and is given by the first term of (2.3). Notice that by Stokes' theorem, it can be calculated in terms of the integral of the curvature of the connection over an area enclosed by the closed curve on the base.

In the event that the momentum is not zero, the system experiences a steady drift to the motion caused by the internal shape changes. The reorientation of this system can always be decomposed into two components: the geometric phase, determined by the shape of the path and the area enclosed by it, and the dynamic phase, driven by the internal kinetic energy of the system characterized by the momentum.

For general systems where $G_{\mu}$ is not abelian, there are still some work to do to clarify the relation between equation (2.2) and the formulations in Marsden, Montgomery and Ratiu [1990]. A fuller understanding of this relationship will help to lay a better foundation for nonholonomic phases.

Indeed, things get even more interesting and also harder to analyse when the system has both rolling constraints and symmetry. As brought out earlier (see equations (1.1) and (1.2)), [BKMM] has developed a framework for studying such systems and has used an interesting example, the snakeboard, to illustrate this theory. It shows that for the snakeboard, while its momentum is not conserved, there is a particular component of its angular momentum, called nonholonomic momentum, $p$, that satisfies a special equation

$$
\dot{p}=\dot{r}^{T} H(r) \dot{r}+\dot{r}^{T} K(r) p+p^{T} D(r) p .
$$


Equation (2.2) remains valid if one uses the nonholonomic momentum and nonholonomic connection. Since the momentum equation (2.4) has no explicit $g$ dependence, when one has a given internal motion, this equation can be solved for $p$ and from it, the attitude and position of the snakeboard can be calculated by an integration using equation (2.2). This strategy parallels that used for the planar $N$-body and $3 D$ rigid body.

While computer simulations on the snakeboard and the roller racer (see Tsakiris [1995]) using periodic inputs have provided us some insights, we do not yet have a complete geometric understanding of the phases for such systems. In general, the net displacement of the mechanism produced by periodic inputs is the geometric phase, or holonomy, associated with the connection. In the case of snakeboard, however, the net displacement is a non-trivial combination of the geometric and dynamic phases. Understanding the increased complexity of the relationship between geometric and dynamic phases for this class of systems is the subject of current research.

\section{References}

Bates, L. and J. Sniatycki [1993] Nonholonomic reduction, Rep. Math. Phys. 32, 99-115.

Bloch, A.M., P.S. Krishnaprasad, J.E. Marsden, and R. Murray [1996] Nonholonomic mechanical systems with symmetry. Arch. Rat. Mech. An., 136, 21-99.

Bloch, A.M., P.S. Krishnaprasad, J.E. Marsden, and G. Sánchez de Alvarez [1992] Stabilization of rigid body dynamics by internal and external torques, Automatica 28, 745-756.

Bloch, A.M., N. Leonard and J.E. Marsden [1997] Stabilization of Mechanical Systems Using Controlled Lagrangians, $C D C, 1997$.

Getz, N.H. and J.E. Marsden [1995] Control for an autonomous bicycle, Intern. Conf. on Rob. and Auto., IEEE, Nagoya, Japan, May, 1995.

Koon, W.S. [1997] Reduction, Reconstruction and Optimal Control for Nonholonomic Mechanical Systems with Symmetry. Ph.D. thesis, Department of Mathematics, UC Berkeley.

Koon, W.S. and J.E. Marsden [1997a] Optimal control for holonomic and nonholonomic mechanical systems with symmetry and Lagrangian reduction. SIAM J. Cont. and Optim. 35, 901-929.

Koon, W.S. and J.E. Marsden [1997b] The Hamiltonian and Lagrangian Approaches to the Dynamics of Nonholonomic Systems. Rep. Math. Phys., to appear.
Koon, W.S and J.E. Marsden [1997c] Poisson Reduction for Nonholonomic Mechanical Systems with Symmetry. preprint.

Leonard, N.E. and J.E. Marsden [1997] Stability and drift of underwater vehicle dynamics: Mechanical systems with rigid motion symmetry, Physica $D$ 105, 130-162.

Marsden, J.E. [1992], Lectures on Mechanics London Mathematical Society Lecture note series. 174, Cambridge Univ. Press.

Marsden, J.E., R. Montgomery, and T.S. Ratiu [1990] Reduction, symmetry, and phases in mechanics. Mem. AMS 436.

Marsden, J.E. and J. Ostrowski [1997] Symmetries in Motion: Geometric Foundations of Motion Control (to appear).

Marsden, J.E. and T.S. Ratiu [1994] Symmetry and Mechanics. Texts in Appl. Math., 17, Springer.

Marsden, J.E. and J. Scheurle [1993b] The reduced Euler Lagrange equations, Fields Inst. Comm. 1, 139-164.

Murray, R.M. [1995] Nonlinear control of mechanical systems: A Latgrangian perspective. In IFAC Symposium on Nonlinear Control Systems Design (NOLCOS), pages 378-389, Tahoe City.

Ostrowski, J., J.P. Desai, and V. Kumar [1997] Optimal gait selection for nonholonomic locomotion systems, $C D C, 1997$.

Simo, J.C., D.R. Lewis, and J.E. Marsden [1991] Stability of relative equilibria I: The reduced energy momentum method, Arch. Rat. Mech. Anal. 115, 15-59.

Tsakiris, D.P. [1995] Motion Control and Planning for Nonholonomic Kinematic Chains. Ph.D. thesis, Univ. of Maryland.

van der Schaft, A.J. \& B.M. Maschke [1994] On the Hamiltonian formulation of nonholonomic mechanical systems, Rep. Math. Phys. 34, 225-233.

Zenkov, D.V., A.M. Bloch, and J.E. Marsden [1997] The Energy Momentum Method for the Stability of Nonholonomic Systems preprint. 